\title{
Magnetic-field annihilation and island formation in electron-scale current sheet in Earth's magnetotail
}

Hiroshi Hasegawa ( $\square$ hase@stp.isas.jaxa.jp )

Institute of Space and Astronautical Science, JAXA https://orcid.org/0000-0002-1172-021X

Richard Denton

Department of Physics and Astronomy, Dartmouth College

Kevin Genestreti

Southwest Research Institute

Takuma Nakamura

Space Research Institute, Austrian Academy of Sciences

Tai Phan

Space Sciences Laboratory

Rumi Nakamura

Space Research Institute

Kyoung-Joo Hwang

Southwest Research Institute

Narges Ahmadi

Laboratory for Atmospheric and Space Physics, University of Colorado

Quanqi Shi

Shandong Provincial Key Laboratory of Optical Astronomy and Solar-Terrestrial Environment, Institute of Space Science, Shandong University, Weihai, Shandong

Michael Hesse

Space Plasma Physics Group, University of Bergen

James Burch

Southwest Research Institute https://orcid.org/0000-0003-0452-8403

James Webster

Rice University

\section{Roy Torbert}

University of New Hampshire https://orcid.org/0000-0001-7188-8690

\section{Barbara Giles}

NASA Goddard Space Flight Center

\section{Daniel Gershman}

NASA Goddard Space Flight Center

Christopher Russell

Department of Earth, Planetary and Space Sciences, University of California, Los Angeles 


\section{Robert Strangeway}

Institute of Geophysics and Planetary Physics, UCLA

\section{P.-A. Lindqvist}

Royal Institute of Technology https://orcid.org/0000-0001-5617-9765

\section{Yuri Khotyaintsev}

Swedish Institute of Space Physics https://orcid.org/0000-0001-5550-3113

\section{Robert Ergun}

University of Colorado, Boulder

\section{Yoshifumi Saito}

JAXA

\section{Article}

Keywords: magnetic-to-particle energy conversion, electron-scale diffusion region, EDR, magnetic-field annihilation, island formation

Posted Date: July 30th, 2020

DOI: https://doi.org/10.21203/rs.3.rs-40743/v1

License: (c) (i) This work is licensed under a Creative Commons Attribution 4.0 International License. Read Full License 
1 Magnetic-field annihilation and island formation in electron-scale current sheet in

2 Earth's magnetotail

3 H. Hasegawa ${ }^{1 *}$, R. E. Denton ${ }^{2}$, K. J. Genestreti ${ }^{3}$, T. K. M. Nakamura ${ }^{4}$, T. D. Phan ${ }^{5}$, R. 4 Nakamura $^{4}$, K.-J. Hwang ${ }^{6}$, N. Ahmadi ${ }^{7}$, Q. Q. Shi ${ }^{8}$, M. Hesse ${ }^{9}$, J. L. Burch ${ }^{6}$, J. M. 5 Webster $^{6}$, R. B. Torbert ${ }^{10,3}$, B. L. Giles ${ }^{11}$, D. J. Gershman ${ }^{11}$, C. T. Russell ${ }^{12}$, R. J. 6 Strangeway $^{12}$, P.-A. Lindqvist ${ }^{13}$, Y. V. Khotyaintsev ${ }^{14}$, R. E. Ergun ${ }^{15}$, and Y. Saito ${ }^{1}$

$7{ }^{1}$ Institute of Space and Astronautical Science, Japan Aerospace Exploration Agency, 8 Sagamihara, Japan.

$9 \quad{ }^{2}$ Department of Physics and Astronomy, Dartmouth College, Hanover, NH, USA.

$10 \quad{ }^{3}$ Southwest Research Institute, Durham, NH, USA.

$11{ }^{4}$ Space Research Institute, Austrian Academy of Sciences, Graz, Austria.

$12 \quad{ }^{5}$ Space Sciences Laboratory, University of California, Berkeley, CA, USA.

$13{ }^{6}$ Southwest Research Institute, San Antonio, TX, USA.

$14{ }^{7}$ Laboratory for Atmospheric and Space Physics, University of Colorado, Boulder, CO, USA.

$15{ }^{8}$ Shandong Provincial Key Laboratory of Optical Astronomy and Solar-Terrestrial 16 Environment, Institute of Space Sciences, Shandong University, Weihai, China.

17 9Space Plasma Physics Group, University of Bergen, Bergen, Norway.

$18{ }^{10}$ Physics Department, University of New Hampshire, Durham, NH, USA.

$19{ }^{11}$ NASA Goddard Space Flight Center, Greenbelt, MD, USA.

$20{ }^{12}$ Department of Earth, Planetary, and Space Sciences, University of California, Los Angeles, 21 CA, USA.

${ }^{13}$ Royal Institute of Technology, Stockholm, Sweden.

$23{ }^{14}$ Swedish Institute of Space Physics, Uppsala, Sweden.

$24{ }^{15}$ Department of Astrophysical and Planetary Sciences, University of Colorado, Boulder, CO, 25 USA.

26 *e-mail: hase@stp.isas.jaxa.jp 


\section{Summary}

Establishing the mechanism of magnetic-to-particle energy conversion through magnetic reconnection in current sheets ${ }^{1}$ is the key to understanding the impact of fast release of magnetic energy in many space and astrophysical plasma systems, such as during magnetospheric substorms ${ }^{2,3}$. It is generally believed that an electron-scale diffusion region (EDR), where a magnetic-to-electron energy conversion occurs, has an X-type magnetic-field geometry ${ }^{4}$ around which the energy of anti-parallel magnetic fields injected is mostly converted to the bulk-flow energy of electrons by magnetic tension of reconnected field-lines ${ }^{5,6}$. However, it is at present unknown exactly how this energy conversion occurs in EDRs, because there has been no observational method to fully address this problem. Here we present state-of-the-art analysis of multi-spacecraft observations in Earth's magnetotail of an electron-scale current sheet, which demonstrates that contrary to the standard model of reconnection with an X-type EDR geometry, the fast energy conversion in the detected EDR was caused mostly by magneticfield annihilation, rather than reconnection. Furthermore, we detected a magnetic island forming in the EDR itself, implying that the EDR had an elongated shape ideal for island generation $^{7}$ and magnetic-field annihilation. The experimental discovery of the annihilation-dominated EDR reveals a new form of energy conversion in the reconnection process that can occur when the EDR has evolved from the X-type to planar geometry.

\section{Main text}

Magnetic reconnection in Earth's magnetotail is fast, with an inflow plasma speed of $\sim 0.1$ times the inflow Alfvén speed, and has large-scale impacts, for example, on explosive release of magnetic energy during magnetospheric substorms ${ }^{2,3}$. Magnetotail reconnection occurs under such simple boundary conditions that on the two sides of the current sheet the magnetic fields are approximately oppositely-directed with a comparable intensity, and plasmas are laminar with similar densities and temperatures. Such nearly anti-parallel and symmetric reconnection is ideal for studying intrinsic properties of reconnection in collisionless plasmas.

In the standard model of reconnection, the EDR where both ions and electrons violate the 'frozen-in condition' and a magnetic-to-electron energy conversion occurs is localised with an X-type magnetic-field geometry ${ }^{4}$ (Fig. 1a). It is generally accepted that in such EDRs most of the energy of anti-parallel magnetic fields injected by the inflow is partitioned to the bulk-flow energy ${ }^{5,6}$ of electrons ejected into the regions downstream of the EDR, called the exhausts, by magnetic tension (Lorentz force) of reconnected field-lines. Numerical simulations ${ }^{7}$ suggest that the EDR may be elongated to a planar geometry (Fig. 1b) and such a spatially extended EDR can be a site ideal for the generation and growth of small-scale magnetic islands (Fig. $1 \mathrm{c}, \mathrm{d})$. However, evidence for islands forming in the EDR itself has so far been lacking. Moreover, it is unknown exactly how the energy conversion occurs in observed EDRs. This is not only because unambiguous identification of EDR structures in space requires highspatiotemporal-resolution plasma measurements, but also because there has been no dataanalysis method to distinguish whether the magnetic field injected into the EDR is reconnected or annihilated.

Here we present in-depth analysis of the fortuitous multi-spacecraft observations of a magnetotail $\mathrm{EDR}^{8}$, providing direct evidence for a magnetic island forming in an elongated electron-scale current sheet (ECS). Fast four-point measurements of electromagnetic fields and plasmas by the Magnetospheric Multiscale (MMS) mission ${ }^{9}$, combined with novel dataanalysis techniques, demonstrate that fast magnetic-to-particle energy conversion in the detected ECS was dominated by magnetic-field annihilation, rather than reconnection. 
In Fig. 2a-d, we show the context of the reconnecting current sheet observed by the MMS 2 spacecraft on 10 August 2017 at 12:17:40-12:19:40 UT, when MMS was fully embedded in the hot magnetotail plasma sheet ${ }^{8}$ at geocentric solar magnetospheric (GSM) coordinates of $(-15.2,4.6,3.1)$ in units of Earth radii and a weak substorm occurred with a peak Auroral Electrojet index ${ }^{10}$ of $\sim 200 \mathrm{nT}$. The current sheet at 12:18:30 UT is characterized by a reversal from anti-sunward to sunward ion flows (negative to positive $v_{\mathrm{i} x}$ change in Fig. $2 \mathrm{~b}$ ), crossing from its southern to northern side (negative to positive $B_{x}$ change in Fig. 2a). The ion flow speeds around the start and end of the interval are comparable to the ion Alfvén speed $V_{\mathrm{iA}}=$ $B /\left(\mu_{0} \rho\right)^{1 / 2} \approx 850 \mathrm{~km} \mathrm{~s}^{-1}$ based on the magnetic field intensity $B \approx 15 \mathrm{nT}$ and proton number density of $0.15 \mathrm{~cm}^{-3}$, where $\mu_{0}$ is the vacuum permeability and $\rho$ is plasma mass density. A fast dawnward electron flow $\left(v_{\mathrm{e} y} \approx-10 \mathrm{Mm} \mathrm{s}^{-1}\right.$ in Fig. $\left.2 \mathrm{c}\right)$ and a relatively slow duskward ion flow with no enhancement at the current sheet show that its electric current was supported by electrons. These features indicate that MMS crossed an ECS embedded inside a region of large-scale reconnection when traversing from its anti-sunward exhaust to sunward exhaust. Earlier studies of this current sheet ${ }^{8,11}$ reported established signatures of EDRs ${ }^{4,9}$, including oppositely-directed electron jets with a speed exceeding $V_{\mathrm{iA}}$ (Fig. 2c), crescent-shaped electron velocity distributions and magnetic-to-particle energy conversion.

We investigate the ECS structure in detail by use of two sophisticated data-analysis techniques (Methods) that can reconstruct multi-dimensional magnetic field structures in regions around the spacecraft from in situ measurements of the magnetic field and plasma bulk parameters (density, velocity, and temperature for ions and electrons). One is a singlespacecraft method based on electron-magnetohydrodynamics (EMHD) equations that can recover quasi-steady, two-dimensional (2-D) magnetic, electrostatic, and electron-velocity fields around the path of the observing spacecraft, hereafter called the EMHD reconstruction ${ }^{12}$. The other is a multi-spacecraft method based on polynomial (second-order Taylor) expansion of the magnetic field that can recover three-dimensional (3-D) magnetic field using instantaneous measurements by the four spacecraft of the magnetic field and particle current density, called the polynomial reconstruction ${ }^{13}$.

Figure 2e-k shows the magnetic field and plasma data from MMS used as input for the two reconstruction techniques, in a current-sheet ('LMN') coordinate system (Methods): the current-sheet normal points along $\boldsymbol{N}$ (roughly northward in the magnetotail), the reconnecting anti-parallel magnetic field component is along $\boldsymbol{L}$ (roughly sunward), and $\boldsymbol{M}=\boldsymbol{N} \times \boldsymbol{L}$ is along the 'X-line' direction (roughly duskward). A weak guide field $(\sim 2 \mathrm{nT})^{8}$, the component $\left(B_{M}\right)$ along the X-line of the magnetic field external to the entire larger-scale current sheet or, in this case, at the centre of the ECS (Fig. 2f), confirms that reconnection occurred under nearly antiparallel magnetic-field conditions. Negative to positive variations of $B_{N}$ (Fig. $2 \mathrm{~g}$ ) is consistent with MMS moving from the anti-sunward to sunward side of the reconnection site; the X-line was moving anti-sunward (Methods). The assumptions of constant density and constant and isotropic electron temperature made in the EMHD reconstruction are approximately satisfied for an intense current-density interval at 12:18:32.1-12:18:34.0 UT (Fig. 2i,k), to which the method is applied. The spacecraft separation $\sim 18 \mathrm{~km}$ was comparable to the electron inertial length $\left(d_{\mathrm{e}} \sim 14 \mathrm{~km}\right)$, allowing us to determine the ECS structure.

Both the EMHD and polynomial reconstruction results (Fig. 3a-d, f-i) clearly show that an electron-scale magnetic island was forming and growing in the ECS with a thickness of about one $d_{\mathrm{e}}$. Figure 3a-d suggests that over a $\sim 0.4 \mathrm{~s}$ interval of 12:18:32.6-12:18:33.0 UT, during which the current sheet was crossed in the order of MMS 1, MMS 3, MMS 4, and MMS 2 (Fig. 2e), both the length (along $\boldsymbol{L}$ ) and width (along $\boldsymbol{N}$ ) of the island grew. An entirely consistent feature is seen in Fig. 3f-i. Furthermore, the energy-conversion rate $\boldsymbol{j} \cdot \boldsymbol{E}^{\prime}=\boldsymbol{j}_{\mathrm{p}} \cdot(\boldsymbol{E}+$ 
$\left.\boldsymbol{v}_{\mathrm{e}} \times \boldsymbol{B}\right)^{14}$ (Fig. 3e), where the particle current density is $\boldsymbol{j}_{\mathrm{p}}=e n_{\mathrm{e}}\left(\boldsymbol{v}_{\mathrm{i}}-\boldsymbol{v}_{\mathrm{e}}\right)$ with the elementary charge of $e, \boldsymbol{E}$ the electric field measured in the spacecraft frame and $\boldsymbol{B}$ the magnetic field, is strongly positive around an X-point closer to the centre of the reconstruction domain. Its magnitude $\left(\sim 0.5 \mathrm{nW} \mathrm{m}^{-3}\right)$ is comparable to an expected value $\left(\sim 0.2 \mathrm{nW} \mathrm{m}^{-3}\right)$ based on the current density $\sim 150 \mathrm{nA} \mathrm{m}{ }^{-2}$ (Fig. $2 \mathrm{~h}$ ) and reconnection electric field $\sim 1.3$ $\mathrm{mV} \mathrm{m}^{-1}$ expected for $B \approx 15 \mathrm{nT}$ and inflow ion speed of $\sim 0.1 V_{\mathrm{iA}} \approx 85 \mathrm{~km} \mathrm{~s}^{-1}$ (Fig. 2b). We thus conclude that reconnection at the X-point was ongoing. Note that the active X-point was captured inside the MMS tetrahedron during the ECS crossing (Fig. 3a-d,g,h), reinforcing the conclusions based on the reconstruction results. Our multi-spacecraft analysis also shows that the encountered ECS was elongated in the exhaust $(\boldsymbol{L})$ direction (Methods).

In order to reveal how fast the magnetic island was growing, we calculated the amount of magnetic flux per unit length along $\boldsymbol{M}$ embedded between the reconstructed X- and O-points closer to the centre of the reconstruction domain. Consistent with the island growth, the flux increases with time (Fig. 4a) and the measured component $\left(E_{M}\right)$ along $\boldsymbol{M}$ of the electric field appears to vary in space and time (Fig. 4c). However, the estimated reconnection electric fields (Fig. 4b) are about one order of magnitude smaller than the measured $E_{M}$ that is comparable to the expected value of $\sim 1.3 \mathrm{mV} \mathrm{m}^{-1}$. This result, surprisingly, suggests that the in-plane magnetic field injected into the ECS was mostly annihilated, rather than reconnected, at the time and portion of the ECS observed by MMS.

Another observational signature consistent with the annihilation-dominated EDR is the electron outflow speed ( 3,000 $\mathrm{km} \mathrm{s}^{-1}$ ) (Fig. $2 \mathrm{j}$ and Extended Data Fig. $2 \mathrm{~b}$ ) much lower than the electron Alfvén speed $\left(V_{\mathrm{eA}} \approx 17 \mathrm{Mm} \mathrm{s}^{-1}\right)$ based on $B_{L}=7 \mathrm{nT}$ outside of the ECS (Fig. 2e) and $n_{\mathrm{e}}=0.15 \mathrm{~cm}^{-3}$ (Fig. 2i). It is possible that magnetic tension resulting under a low reconnection rate $\left(E_{\text {Ree }}\right.$ of order $0.1 \mathrm{mV} \mathrm{m}^{-1}$ ) (Fig. $\left.4 \mathrm{~b}\right)$ was not strong enough to accelerate electrons, injected into the ECS at a high rate (equivalent to $E_{\mathrm{str}, M}=1-2 \mathrm{mV} \mathrm{m}^{-1}$ ) (Fig. $4 \mathrm{c}$ ), to a speed comparable to $V_{\mathrm{eA}}$.

Is fast annihilation of the magnetic field as detected by MMS physically possible in an ECS? For quasi-steady 2-D reconnection in collisionless plasmas, electron unmagnetisation (violation of the electron frozen-in condition) at the X-line occurs when off-diagonal terms of the electron pressure tensor are significant ${ }^{15}$. Consistently, non-gyrotropic electron velocity distributions as a manifestation of electron unmagnetisation have been observed in the present ${ }^{8}$ as well as other EDRs ${ }^{4,9}$, and earlier studies demonstrated that they can quantitatively account for the electric field $\left(E_{M}\right)$ of fast reconnection as observed ${ }^{16,17}$. We derive a diffusion equation for $B_{L}$ (equation (4) in Methods),

$$
\frac{\partial B_{L}}{\partial t} \approx r_{\mathrm{ge}} V_{\infty} \frac{\partial^{2} B_{L}}{\partial N^{2}}
$$

where $r_{\mathrm{ge}}$ is the gyroradius of thermal electrons and $V_{\infty}$ is the electron inflow speed $\left(\left|v_{\mathrm{e} N}\right|\right)$ immediately outside of the EDR, that is applicable when the generalized Ohm's law has a term equivalent to the non-gyrotropic electron pressure tensor and the ECS is elongated in the exhaust direction. This equation is supported by a solution of the EMHD equations and is consistent with the observed magnetic-field annihilation (Methods). The fast annihilation is in stark contrast with the classic (resistive magnetohydrodynamics) model ${ }^{18,19}$ of reconnection in an elongated current sheet in which magnetic-field annihilation is negligibly small under magnetotail conditions. However, we do not exclude a likely possibility that at around the sunward and anti-sunward ends of the present ECS, where the magnetic field may have a Ytype geometry (Fig. 1b,c), the magnetic field was efficiently reconnected and ejected downstream, as observed for other magnetotail reconnection events ${ }^{20}$. 
The approximately planar ECS, electron-scale island forming in the EDR itself and annihilation-dominated energy conversion, detected in this study, all support the existence of an EDR elongated in the exhaust direction (Fig. 1c) that is favourable for the island generation? The detection of an island forming in such an EDR suggests that MMS serendipitously encountered the very initial stage of the island growth, so that the growth rate may be low (Fig. $4 \mathrm{c})$. The present observations also suggest that magnetic island formation is an intrinsic process in the EDR of anti-parallel reconnection, supporting the conclusion from a kinetic simulation? rather than other simulation studies that predicted that an island may form in ECSs only when the guide field is $\operatorname{large}^{21}$. Furthermore, the experimental discovery of magnetic-field annihilation in a reconnecting ECS provides the first direct confirmation that magnetic energy can be irreversibly dissipated through collisionless reconnection ${ }^{22}$. Magnetic island formation and magnetic-field annihilation are key ingredients for the generation ${ }^{23,24}$ and dissipation, respectively, of plasma turbulent energy and could play a role in electron acceleration ${ }^{25-27}$. These aspects and the role played by magnetic-field annihilation in electron heating will need to be quantitatively assessed in the future, but our finding reveals a new form of magnetic-toparticle energy conversion in the reconnection process that can occur when the EDR has evolved from the X-type to planar geometry.

\section{Data availability}

The entire MMS dataset is publicly available at https://lasp.colorado.edu/mms/sdc/public/.

\section{Online content}

Methods, along with any additional Extended Data figures and references and Source Data, are available in the online version of the paper.

\section{References}

1. Yamada, M., Kulsrud, R. \& Ji, H. Magnetic reconnection. Rev. Mod. Phys., 82, 603-664 (2010).

2. Angelopoulos, V., McFadden, J. P., Larson, D. et al. Tail reconnection triggering substorm onset. Science, 321, 931-935 (2008).

3. Nagai, T., Shinohara, I., Fujimoto, M., Matsuoka, A., Saito, Y., \& Mukai, T. Construction of magnetic reconnection in the near-Earth magnetotail with Geotail. J. Geophys. Res., 116, A04222 (2011).

4. Torbert, R. B., Burch, J. L., Phan, T. D. et al. Electron-scale dynamics of the diffusion region during symmetric magnetic reconnection in space. Science, 362, 1391-1395 (2018).

5. Cassak, P. A. \& Shay M. A. Scaling of asymmetric magnetic reconnection: General theory and collisional simulations, Phys. Plasmas, 14, 102114 (2007).

6. Shay, M. A., Drake, J. F. \& Swisdak, M. Two-scale structure of the electron dissipation region during collisionless magnetic reconnection. Phys. Rev. Lett., 99, 155002 (2007).

7. Daughton, W., Scudder, J. \& Karimabadi, H. Fully kinetic simulations of undriven magnetic reconnection with open boundary conditions. Phys. Plasmas, 13, 072101 (2006).

8. Zhou, M., Deng, X. H., Zhong, Z. H. et al. Observations of an electron diffusion region in symmetric reconnection with weak guide field. Astrophys. J., 870, 34 (2019).

9. Burch, J. L., Torbert, R. B., Phan, T. D. et al. Electron-scale measurements of magnetic reconnection in space. Science, 352, aaf2939 (2016).

10. Davis, T. N. \& Sugiura, M. Auroral electrojet activity index $A E$ and its universal time variations. J. Geophys. Res., 71, 785-801 (1966). 
11. Li, X., Wang, R., Lu, Q. et al. Observation of nongyrotropic electron distribution across the electron diffusion region in the magnetotail reconnection. Geophys. Res. Lett., 46, 14263-14273 (2019).

12. Sonnerup, B. U. Ö., Hasegawa, H., Denton, R. E. \& Nakamura, T. K. M. Reconstruction of the electron diffusion region. J. Geophys. Res. Space Physics, 121, 4279-4290 (2016).

13. Denton, R. E., Torbert, R. B., Hasegawa, H. et al. Polynomial reconstruction of the reconnection magnetic field observed by multiple spacecraft. J. Geophys. Res. Space Physics, 125, e2019JA027481 (2020).

14. Zenitani, S., Hesse, M., Klimas, A. \& Kuznetsova, M. New measure of the dissipation region in collisionless magnetic reconnection. Phys. Rev. Lett. 106, 195003 (2011).

15. Hesse, M., Neukirch, T., Schindler, K., Kuznetsova, M. \& Zenitani, S. The diffusion region in collisionless magnetic reconnection. Space Sci. Rev., 160, 3-23 (2011).

16. Nakamura, R., Genestreti, K. J., Nakamura, T. K. M. et al. Structure of the current sheet in the 11 July 2017 Electron Diffusion Region event. J. Geophys. Res. Space Physics, 124, 1173-1186 (2019).

17. Egedal, J., Ng, J., Le, A. et al. Pressure tensor elements breaking the frozen-in law during reconnection in Earth's magnetotail. Phys. Rev. Lett., 123, 225101 (2019).

18. Parker, E. N. Sweet's mechanism for merging magnetic fields in conducting fluids. $J$. Geophys. Res., 62, 509-520 (1957).

19. Sweet, P. A. in Electromagnetic Phenomena in Cosmical Physics (ed. Lehnert, B.) Cambridge, 123-134 (1958).

20. Nakamura, T. K. M., Genestreti, K. J., Liu, Y.-H. et al. Measurement of the magnetic reconnection rate in the Earth's magnetotail. J Geophys. Res. Space Physics, 123, 91509168 (2018).

21. Drake, J. F., Swisdak, M., Schoeffler, K. M., Rogers, B. N. \& Kobayashi, S. Formation of secondary islands during magnetic reconnection. Geophys. Res. Lett., 33, L13105 (2006).

22. Phan, T. D., Eastwood, J. P., Shay, M. A. et al. Electron magnetic reconnection without ion coupling in Earth's turbulent magnetosheath. Nature, 557, 202-206 (2018).

23. Daughton, W., Roytershteyn, V., Karimabadi, H. et al. Role of electron physics in the development of turbulent magnetic reconnection in collisionless plasmas. Nat. Phys., 7, 539-542 (2011).

24. Franci, L., Cerri, S. S., Califano, F. et al. Magnetic reconnection as a driver for a sub-ionscale cascade in plasma turbulence. Astrophys. J. Lett., 850, L16 (2017).

25. Drake, J. F., Swisdak, M., Che, H. \& Shay, M. A. Electron acceleration from contracting magnetic islands during reconnection. Nature, 443, 553-556 (2006).

26. Chen, L.-J., Bhattacharjee, A., Puhl-Quinn, P. A. et al. Observation of energetic electrons within magnetic islands. Nat. Phys., 4, 19-23 (2008).

27. Wang, R., Lu, Q., Du, A. \& Wang, S. In situ observations of a secondary magnetic island in an ion diffusion region and associated energetic electrons. Phys. Rev. Lett., 104, 175003 (2010).

28. Dunlop, M. W., Balogh, A., Glassmeier, K.-H. \& Robert, P. Four-point Cluster application of magnetic field analysis tools: The Curlometer. J. Geophys. Res., 107, 1384 (2002).

\section{Acknowledgments}

We are grateful for the dedicated efforts of the MMS team. This work was supported by a NASA grant (80NSSC19K0254) and the Austrian Research Fund (FWF) P32175-N27. H.H. thanks W. Daughton for discussions. 


\section{Author contributions}

H.H. and R.E.D. carried out the data analysis and interpretation, theoretical analysis and manuscript preparation. K.J.G., T.K.M.N., T.D.P., R.N., K.J.H., N.A., and J.M.W. contributed to the data interpretation and manuscript preparation. Q.Q.S. contributed to the data analysis and interpretation. M.H. contributed to the theoretical analysis. J.L.B. led the successful design and operation of the MMS mission and contributed to the data interpretation. R.B.T., P.A.L., Y.V.K., and R.E.E. contributed to the development and operation of the electric-field experiments. B.L.G., D.J.G. and Y.S. contributed to the development and operation of and the interpretation of data from the Fast Plasma Investigation instruments. C.T.R. and R.J.S. contributed to the development and operation of the fluxgate magnetometers.

\section{Competing interests}

The authors declare no competing interests.

\section{Additional information}

Correspondence and requests for materials should be addressed to H.H.
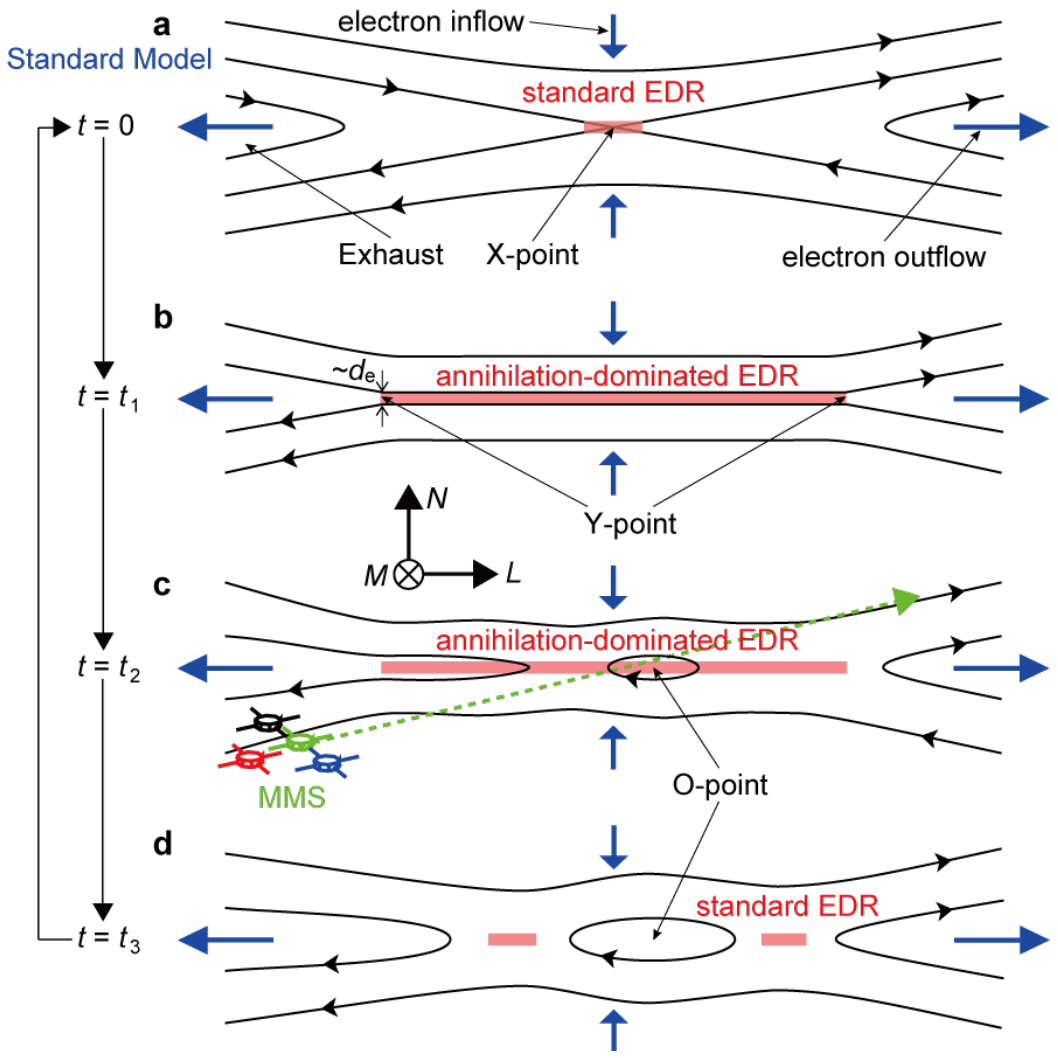

Fig. 1 | Standard picture of the reconnection region and possible sequence of time evolution of the observed electron-scale current sheet. a, Standard EDR with an X-type magnetic-field geometry where the magnetic-to-particle energy conversion is mostly due to reconnection ${ }^{4}$. b, EDR after elongation along $\boldsymbol{L}$, as seen in simulation ${ }^{7}$, where the energy conversion may be mostly due to magnetic-field annihilation (Methods). c, Initial stage of the magnetic-island formation in the EDR, as observed by MMS. d, Possible later stage of the island growth in exhausts of the EDRs where the energy conversion is again dominated by reconnection. 


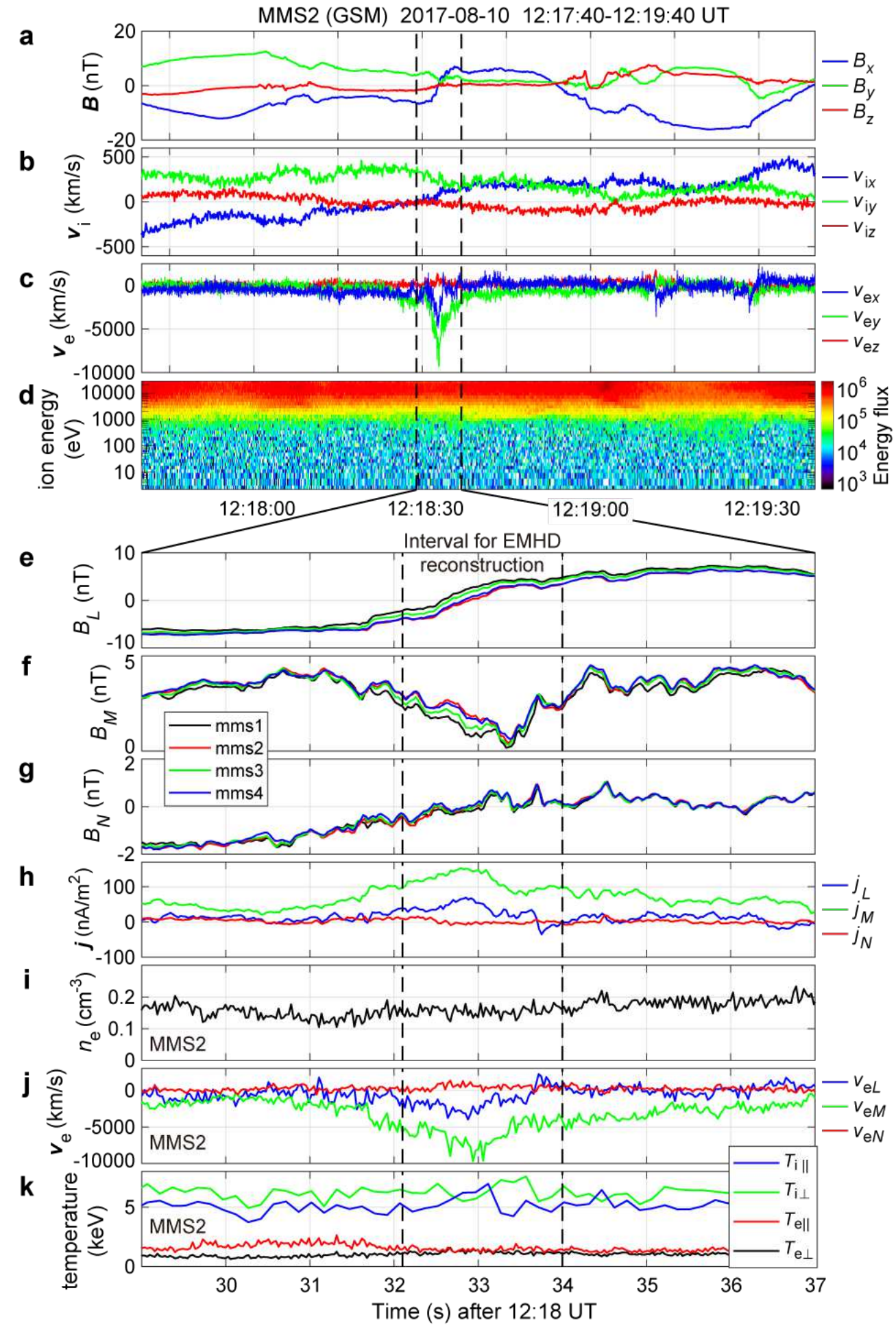

Fig. 2 | Overview of MMS observations of an ECS with both ion and electron reconnection jet signatures. a-d, GSM components of the magnetic field $\left(\mathbf{a} ; B_{x}, B_{y}\right.$ and $\left.B_{z}\right)$, ion velocity $\left(\mathbf{b} ; v_{\mathrm{i} x}, v_{\mathrm{i} y}\right.$ and $\left.v_{\mathrm{i} z}\right)$, electron velocity $\left(\mathbf{c} ; v_{\mathrm{e} x}, v_{\mathrm{e} y}\right.$ and $\left.v_{\mathrm{e} z}\right)$ and ion energy-time spectrogram of omni-directional differential energy flux (d; colour scale, in units of $\mathrm{keV} \mathrm{s}^{-1} \mathrm{~cm}^{-2} \mathrm{sr}^{-1} \mathrm{keV}^{-1}$ ) seen by MMS 2. $\mathbf{e}-\mathbf{h}$, Components in a common current-sheet (LMN) coordinate system of the magnetic field (e-g; $B_{L}, B_{M}$ and $B_{N}$ ) from all four spacecraft (black, MMS 1; red, MMS 2; green, MMS 3; blue, MMS 4) and current density $\boldsymbol{j}=(\nabla \times \boldsymbol{B}) / \mu_{0}{ }^{28}\left(\mathbf{h} ; j_{L}, j_{M}\right.$ and $\left.j_{N}\right)$. $\mathbf{i}-\mathbf{k}$, MMS 2 measurements of the electron density $\left(\mathbf{i} ; n_{\mathrm{e}}\right)$, electron velocity in LMN coordinates $(\mathbf{j}$; $v_{\mathrm{e} L}, v_{\mathrm{e} M}$ and $\left.v_{\mathrm{e} N}\right)$, and ion and electron temperatures $\left(\mathbf{k} ; T_{\mathrm{i}}\right.$ and $\left.T_{\mathrm{e}}\right)$ in the directions parallel (II) and perpendicular $(\perp)$ to the local magnetic field. 

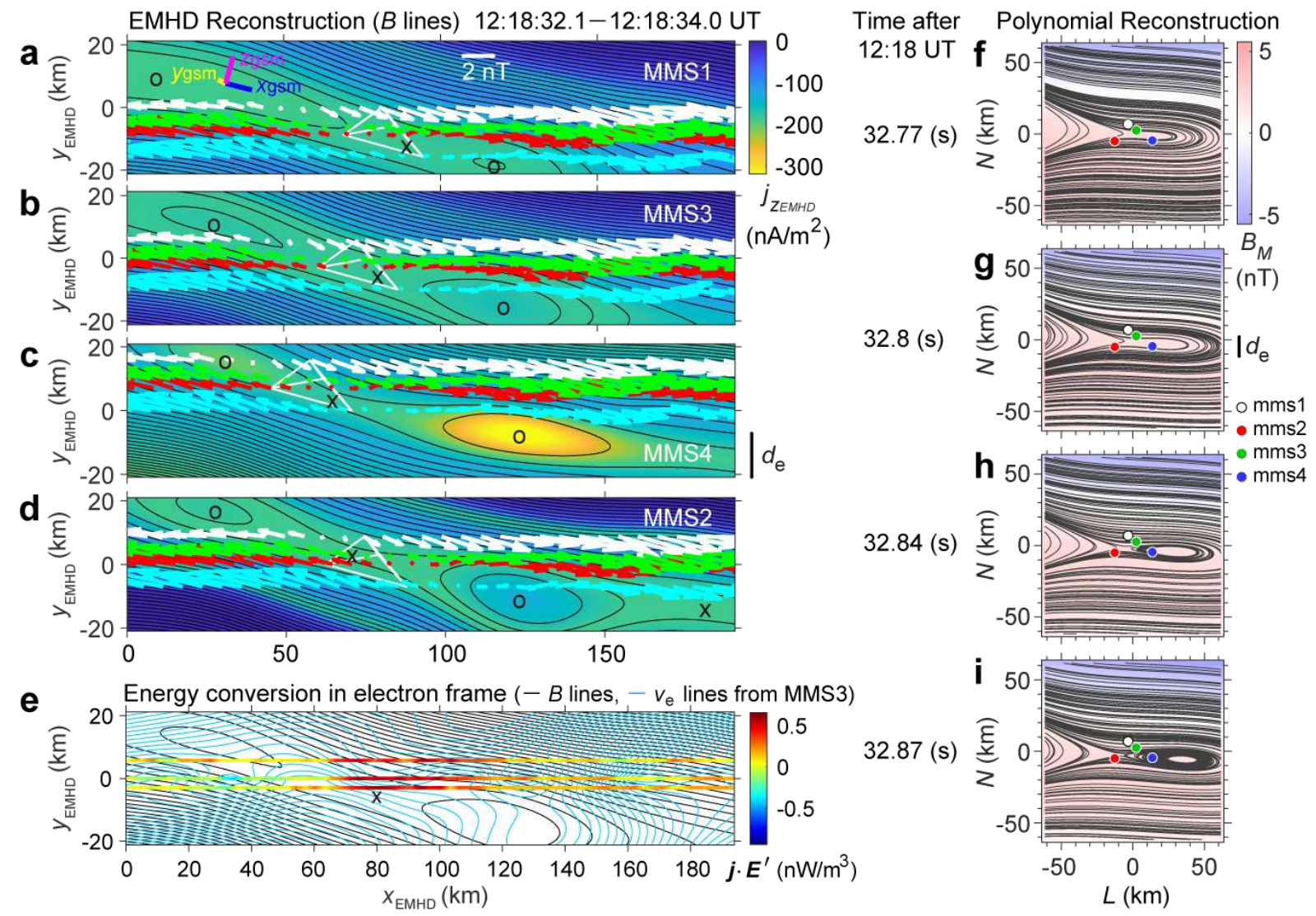

Fig. 3 | Magnetic fields recovered from the two reconstruction methods (Methods). a-d, 2-D magnetic fields from the EMHD reconstruction ${ }^{12}$ using the data taken at 12:18:32.112:18:34.0 UT individually for each of the four spacecraft, shown in the order of current sheet crossing (MMS 1, a; MMS 3, b; MMS 4, c; then MMS 2, d; as seen in Fig. 2e). Black curves show the reconstructed magnetic field-lines, colours the out-of-plane component $\left(j_{z, \mathrm{EMHD}}\right)$ of the reconstructed current density, and arrows the projections onto the reconstruction $\left(x_{\mathrm{EMHD}^{-}}\right.$ $y_{\mathrm{EMHD}}$ ) plane of the measured magnetic fields (white, MMS 1; red, MMS 2; green, MMS 3; 301 cyan, MMS 4) along the paths of the four spacecraft. The bars near the upper-left corner of Fig. $3 \mathrm{a}$ are the projections of the unit GSM axes (blue, $\widehat{\boldsymbol{x}}_{\mathrm{GSM}}$; yellow, $\widehat{\boldsymbol{y}}_{\mathrm{GSM}}$; magenta, $\widehat{\boldsymbol{z}}_{\mathrm{GSM}}$ ). e, Magnetic field-lines (black curves) and electron streamlines (blue curves) reconstructed from the MMS 3 data, with $\boldsymbol{j} \cdot \boldsymbol{E}^{\prime}=\boldsymbol{j}_{\mathrm{p}} \cdot\left(\boldsymbol{E}+\boldsymbol{v}_{\mathrm{e}} \times \boldsymbol{B}\right)^{14}$ measured along the paths of MMS 1, MMS 2 , and MMS 3 in colour. $\mathbf{f}-\mathbf{i}$, Projection onto the $L-N$ plane of 3-D magnetic field-lines reconstructed using the polynomial reconstruction ${ }^{13}$ from instantaneous measurements (time after 12:18 UT: $32.77 \mathrm{~s}, \mathbf{f} ; 32.8 \mathrm{~s}, \mathbf{g} ; 32.84 \mathrm{~s}, \mathbf{h} ; 32.87 \mathrm{~s}, \mathbf{i})$ by the four spacecraft of $\boldsymbol{B}$ and $\boldsymbol{j}_{\mathrm{p}}$,

308 with reconstructed $B_{M}$ in colour. 

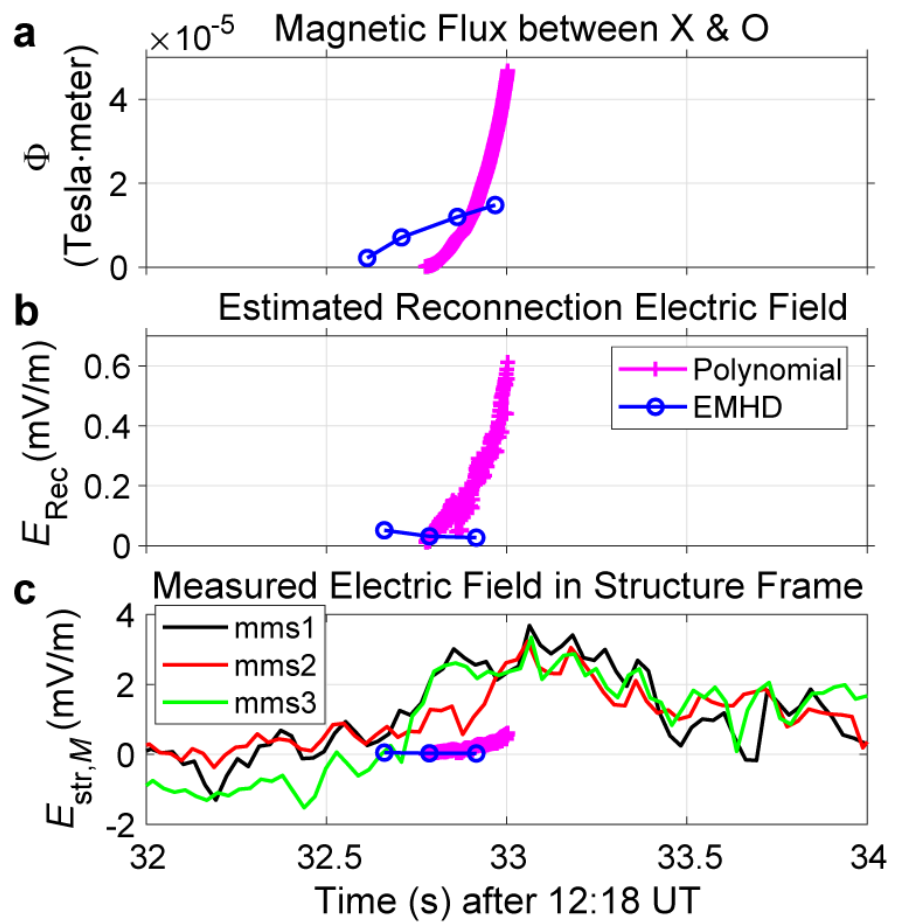

Fig. 4 | Reconnection electric fields, estimated from the EMHD and polynomial reconstructions, compared with the measured electric field. a, In-plane magnetic flux $(\Phi)$ embedded between the X-and O-points around the centre of the reconstruction domain as a function of time which is defined as that of the current sheet crossing $\left(B_{L}=0\right)$ for the EMHD reconstruction. $\mathbf{b}$, Reconnection electric field $\left(E_{\mathrm{Rec}}=\partial \Phi / \partial t\right)$ estimated from the temporal rate of change in $\Phi$. c, $M$ component $\left(E_{\mathrm{str}, M}\right)$ of the electric field from MMS 1, MMS 2, and MMS 3 that made reliable electric field measurements, transformed into the frame comoving with the structure (Methods), along with $E_{\mathrm{Rec}}$. 


\section{Methods}

\section{Data handling.}

For this study, MMS burst-mode data from the following instrument suites were used: FIELDS $^{29}$, including the electric-field instruments ${ }^{30,31}$ and magnetometers ${ }^{32}$, and FPI (electron and ion plasma distributions and moments) ${ }^{33}$. All data are from the standard level-2 data products, which are publicly available at the MMS Science Data Center (https://lasp.colorado.edu/mms/sdc/public).

\section{Current-sheet (LMN) coordinate system.}

The final LMN coordinate system used in this study, with $\boldsymbol{L}=$ $(0.955,-0.298,-0.021)_{\mathrm{GSM}} \quad, \quad \boldsymbol{M}=(0.296,0.953,-0.059)_{\mathrm{GSM}}, \quad$ and $\boldsymbol{N}=$ $(0.038,0.050,0.998)_{\mathrm{GSM}}$, was determined through optimization of the EMHD reconstruction results, as detailed below. A preliminary LMN coordinate system used as trial LMN axes in the reconstruction was determined by a hybrid method $^{34}$, which combines the Minimum Variance Analysis (MVA) ${ }^{35}$ and Maximum Directional Derivative (MDD) method ${ }^{36}$ of the magnetic field, applied to four-spacecraft measurements of the magnetic field at 12:18:3112:18:36 UT. The resulting $\mathrm{LMN}$ axes are: $\boldsymbol{L}_{\mathrm{p}}=(0.992,-0.127,-0.022)_{\mathrm{GSM}}, \boldsymbol{M}_{\mathrm{p}}=$ $(0.127,0.992,-0.003)_{\mathrm{GSM}}$, and $\boldsymbol{N}_{\mathrm{p}}=(0.022,-0.000,1.000)_{\mathrm{GSM}}$, which are not very different from the final axes. Results from the MDD method are presented in Extended Data Fig. 1, which shows that the current sheet was roughly planar locally (Extended Data Fig. 1f), satisfying the assumption underlying the MVA, and that the current-sheet normal was stably northward (Extended Data Fig. 1g).

\section{Frame velocity.}

The frame velocity comoving with the structure was estimated by a multi-spacecraft dataanalysis technique known as the spatiotemporal difference (STD) method ${ }^{37}$, applied to smoothed magnetic field data from the four spacecraft at 12:18:32.0-12:18:33.3 UT. The velocity component from the STD method along the minimum magnetic-gradient direction $\boldsymbol{k}_{\text {min }}$, which is often along $\boldsymbol{M}$ (Extended Data Fig. 1i), is usually unreliable ${ }^{38}$ and was not used. The resulting velocity is $\boldsymbol{V}_{\mathrm{STD}}=-100 \boldsymbol{L}_{\mathrm{p}}-28 \boldsymbol{N}_{\mathrm{p}} \mathrm{km} \mathrm{s}^{-1}$, consistent with an anti-sunward moving EDR and south-to-north crossing of the ECS. The normal velocity roughly agrees well with that $\left(-35 \mathrm{~km} \mathrm{~s}^{-1}\right)$ estimated by multi-spacecraft timing analysis ${ }^{8}$. The $L$-component dominated motion of the structure, combined with the approximately planar geometry of the ECS (Extended Data Fig. 1f), indicates that the ECS was elongated in the exhaust direction (Fig. 1b,c). The $L$ and $N$ components of the final structure velocity $\boldsymbol{V}_{\text {str }}=$ $(-30,237,-40)_{\mathrm{GSM}} \mathrm{km} \mathrm{s}^{-1}$, used in the EMHD reconstruction, are the projections along $\boldsymbol{L}$ and $\boldsymbol{N}$ of $\boldsymbol{V}_{\mathrm{STD}}$ and the $M$ component $\left(V_{\mathrm{str}, M}\right)$ is that of the mean ion velocity for the reconstruction interval, so that the electric current is supported mostly by electrons in the structure frame. The electric field transformed into the structure frame is $\boldsymbol{E}_{\mathrm{str}}=\boldsymbol{E}+\boldsymbol{V}_{\text {str }} \times \boldsymbol{B}$.

\section{EMHD reconstruction.}

The EMHD reconstruction ${ }^{12}$ recovers 2-D magnetic, electrostatic, and electron-velocity fields in the reconstruction $\left(x_{\mathrm{EMHD}}-y_{\mathrm{EMHD}}\right)$ plane around the path $\left(y_{\mathrm{EMHD}}=0\right)$ of a single spacecraft from the magnetic field and ion and electron moments data taken by the spacecraft, once a proper LMN coordinate system and frame velocity are defined. The underlying assumptions are that the structure is time-independent in its rest frame $(\partial / \partial t=0)$ and 2-D $\left(\partial / \partial z_{\mathrm{EMHD}}=0\right)$ and has a constant frame velocity $\boldsymbol{V}_{\text {str }}$ (straight spacecraft path), electroninertia terms and ion dynamics can be neglected, and the electron density $n_{\mathrm{e}}$ is constant in space and time. The $x_{\text {EMHD }}$ axis is defined to be anti-parallel to the projection of $\boldsymbol{V}_{\text {str }}$ onto the 
plane perpendicular to $\hat{\boldsymbol{z}}_{\mathrm{EMHD}}=-\boldsymbol{M}$, and $\widehat{\boldsymbol{y}}_{\mathrm{EMHD}}=\hat{\boldsymbol{z}}_{\mathrm{EMHD}} \times \widehat{\boldsymbol{x}}_{\mathrm{EMHD}}$. The generalized Ohm's law (electron momentum equation) used in the reconstruction is thus

$$
\boldsymbol{E}=-\boldsymbol{v}_{\mathrm{e}} \times \boldsymbol{B}-\frac{\nabla \cdot \boldsymbol{P}}{n_{\mathrm{e}} e}
$$

with $\nabla \cdot \boldsymbol{P}=\nabla \tilde{p}+\hat{\boldsymbol{z}}_{\mathrm{EMHD}} f\left(x_{\mathrm{EMHD}}, y_{\mathrm{EMHD}}\right)$ where $\boldsymbol{P}$ is the electron pressure tensor, $\tilde{p}=$ $n_{\mathrm{e}} k_{\mathrm{B}} T_{\mathrm{e}}$ is the part of isotropic electron pressure, which is assumed to be constant and thus $\nabla \tilde{p}=0$. Here $k_{\mathrm{B}}$ is the Boltzmann constant. It has been shown that the term

$$
f\left(x_{\mathrm{EMHD}}, y_{\mathrm{EMHD}}\right)=f(L, N)=n_{\mathrm{e}} \sqrt{2 m_{\mathrm{e}} k_{\mathrm{B}} T_{\mathrm{e}}} \frac{\partial v_{\mathrm{e} L}}{\partial L}
$$

where $m_{\mathrm{e}}$ is electron mass, plays a role equivalent to the effect of off-diagonal terms of the electron pressure tensor for anti-parallel reconnection and can cause the violation of the electron frozen-in condition at the X-point ${ }^{15,39}$. The EMHD reconstruction has been successfully applied to EDR events in both the magnetopause ${ }^{40}$ and magnetotail ${ }^{41}$ current layers.

Extended Data Fig. 2 shows the magnetic field, electron streamlines, and electric field reconstructed for the present ECS using the data from MMS 3, which was located near the centre of the MMS tetrahedron in the reconstruction plane. The final LMN axes were optimized by a multi-spacecraft method ${ }^{40}$, which searches for the invariant-axis $\left(\hat{\mathbf{z}}_{\mathrm{EMHD}}\right)$ orientation that maximizes the correlation coefficient (Extended Data Fig. 2d) between the normalized components of the magnetic field and electron velocity measured by three spacecraft not used as input in the reconstruction and those predicted at points along the paths of the three spacecraft from the reconstructed field maps. The reconstruction axes thus determined are: $\widehat{\boldsymbol{x}}_{\mathrm{EMHD}}=(0.927,-0.271,0.260)_{\mathrm{GSM}}, \widehat{\boldsymbol{y}}_{\mathrm{EMHD}}=(-0.232,0.131,0.964)_{\mathrm{GSM}}$ and $\hat{\boldsymbol{z}}_{\mathrm{EMHD}}=$ $(-0.296,-0.953,0.059)_{\mathrm{GSM}}$. Numerical errors may be large in regions away from the path $\left(y_{\mathrm{EMHD}}=0\right)$ of MMS 3, especially near the four corners of the reconstruction domain. The reconstructed streamlines (Extended Data Fig. 2b) do not clearly show the inflow and outflow patterns as expected for steady reconnection but have a complex feature, probably because of time dependence associated with the growing magnetic island (Fig. 3). However, a quadrupolar pattern of $B_{z, \text { EMHD }}=-B_{M}$ related to the Hall effect ${ }^{42,43}$ is roughly reconstructed.

Extended Data Fig. $2 d$ shows that the magnetic field is well reconstructed with a very high correlation coefficient; we can rely on the reconstructed magnetic fields (Fig. 3a-d). The small $E_{\text {Rec }}$ (Fig. 4) also indicates that the time evolution associated with the island growth was slow, assuring that the assumption of time-independence was approximately satisfied.

\section{Polynomial reconstruction.}

The polynomial reconstruction ${ }^{13,44}$ recovers 3 -D magnetic fields in and around the tetrahedron formed by the four spacecraft from instantaneous measurements by the four spacecraft of the magnetic field and particle current density. Several versions ${ }^{13}$ exist but all are based on second- or third-order Taylor expansion of the magnetic field. It produces a 3-D magnetic field for every moment during a selected time interval, and does not require any assumptions about structural dimensionality, time dependence, and frame velocity. Thus, it is complementary to the physics-based EMHD reconstruction in which steady and 2-D structures are assumed, so that the fields reconstructed using data from a finite time interval, which may violate the model assumptions, may well be time-aliased, while a larger reconstruction domain is allowed.

In this study, we used the 3-D reduced quadratic (RQ-3D) mode ${ }^{13}$ in which less significant parts of the quadratic terms and higher-order terms are neglected. For 3-D fields, the calculation of magnetic flux inside a magnetic island (Fig. 4) is not trivial. The in-plane flux was calculated in two ways, one that first integrates $B_{N}$ from $(L, N)=\left(L_{\mathrm{X}}, N_{\mathrm{X}}\right)$ to $\left(L_{\mathrm{O}}, N_{\mathrm{X}}\right)$ and then adds $B_{L}$ integrated from $\left(L_{0}, N_{\mathrm{X}}\right)$ to $\left(L_{0}, N_{\mathrm{O}}\right)$, and the other that first integrates $B_{L}$ from $(L, N)=$ $\left(L_{\mathrm{X}}, N_{\mathrm{X}}\right)$ to $\left(L_{\mathrm{X}}, N_{\mathrm{O}}\right)$ and then adds $B_{N}$ integrated from $\left(L_{\mathrm{X}}, N_{\mathrm{O}}\right)$ to $\left(L_{0}, N_{\mathrm{O}}\right)$, in the $L-N$ plane 
where the barycentre of the MMS tetrahedron is located. Here, $(L, N)=\left(L_{\mathrm{X}}, N_{\mathrm{X}}\right)$ is the location of the X-point and $\left(L_{O}, N_{\mathrm{O}}\right)$ of the O-point. The two flux values agree very well with each other, which corroborates the validity of the 2-D assumption made in the EMHD reconstruction.

\section{Magnetic-field annihilation due to off-diagonal terms of electron pressure tensor.}

It can be demonstrated that magnetic-field annihilation may occur across $\boldsymbol{N}$ in part extended in the $L$ direction of an ECS with $\partial / \partial M=0$, when the generalized Ohm's law is expressed by equation (1)

$$
\boldsymbol{E}=-\boldsymbol{v}_{\mathrm{e}} \times \boldsymbol{B}+\frac{f(L, N)}{n_{\mathrm{e}} e} \boldsymbol{M} .
$$

We consider Faraday's law in the LMN coordinate system,

$$
\frac{\partial \boldsymbol{B}}{\partial t}=-\nabla \times \boldsymbol{E}=\nabla \times\left(\boldsymbol{v}_{\mathrm{e}} \times \boldsymbol{B}\right)-\frac{1}{n_{\mathrm{e}} e} \nabla f(L, N) \times \boldsymbol{M} .
$$

Here we discuss only the contribution of the second term on the right-hand side (RHS) of equation (3), because the first term does not violate the electron frozen-in condition. Furthermore, only the $L$ component

$$
\frac{\partial B_{L}}{\partial t} \approx \frac{1}{n_{\mathrm{e}} e} \frac{\partial f(L, N)}{\partial N}
$$

is considered, because $B_{N} \approx 0$ in ECSs elongated in the $L$ direction. Since constant electron density, namely incompressible electron fluid $\left(\nabla \cdot v_{\mathrm{e}}=\partial v_{\mathrm{e} L} / \partial L+\partial v_{\mathrm{e} N} / \partial N=0\right)$, can be assumed in $\mathrm{ECSs}^{12,15}$, the following relation results using equation (2)

$$
\frac{\partial B_{L}}{\partial t} \approx-\frac{\sqrt{2 m_{\mathrm{e}} k_{\mathrm{B}} T_{\mathrm{e}}}}{e} \frac{\partial^{2} v_{\mathrm{eN}}}{\partial N^{2}} .
$$

We note that a relation $-B_{\infty} v_{\mathrm{e} N} \approx B_{L} V_{\infty}$ is approximately satisfied inside an EDR because the spatial variations along $N$ of $B_{L}$ and $v_{\mathrm{e} N}$ would be very similar to each other with only sign difference (Extended Data Fig. 3c), while in the inflow region outside of the EDR $B_{L} v_{\mathrm{e} N} \approx$ $E_{0}=B_{\infty} V_{\infty}$ holds for quasi-steady 2-D reconnection ${ }^{45}$. Here $E_{0}$ is the reconnection electric field, and $B_{\infty}$ and $V_{\infty}$ are the $B_{L}$ intensity and the electron inflow speed, respectively, immediately outside of the EDR, with $V_{\infty} \approx 0.1 V_{\mathrm{eA}}$ for fast reconnection where the electron Alfvén speed $V_{\mathrm{eA}}=B_{\infty} /\left(\mu_{0} m_{\mathrm{e}} n_{\mathrm{e}}\right)^{1 / 2}$. Thus, the above relation becomes a diffusion equation for $B_{L}$

$$
\frac{\partial B_{L}}{\partial t} \approx D_{B} \frac{\partial^{2} B_{L}}{\partial N^{2}}
$$

where the diffusion coefficient $D_{B}=r_{\mathrm{ge}} V_{\infty}$ with the electron gyroradius $r_{\mathrm{ge}}=$ $\left(2 m_{\mathrm{e}} k_{\mathrm{B}} T_{\mathrm{e}}\right)^{1 / 2} /\left(e B_{\infty}\right)$. We can therefore conclude that in the presence of the term (2), equivalent to the non-gyrotropic electron pressure tensor term, the in-plane magnetic-field component $B_{L}$ may be annihilated in an EDR. This conclusion is consistent with the theoretical analysis $^{21}$ in which the term (2) was derived by discussing a diffusion of the electron current density $j_{\mathrm{e} M}$ that implies the annihilation of $B_{L}$. On the other hand, since the second term on the RHS of equation (3) has no $M$ component, no annihilation of $B_{M}$ (Hall magnetic field) occurs for 2-D reconnection. Moreover, the form of $D_{B}$ indicates that in principle fast magnetic-field annihilation may occur when fast inflow of the in-plane magnetic flux exists. Notably, an experimental estimate of the diffusion coefficient $D \approx \delta V_{\infty}=d_{\mathrm{e}} E_{\mathrm{str}, M} / B_{\infty} \sim 10^{10} \mathrm{~m}^{2} \mathrm{~s}^{-1}$ for the observed ECS with a thickness $\delta \approx d_{\mathrm{e}}$ and electron beta $\beta_{\mathrm{e}}=r_{\mathrm{ge}}{ }^{2} / d_{\mathrm{e}}{ }^{2} \sim 5$ is comparable to $D_{B}$. An exact solution of the EMHD equations with the dissipation term (2) (Extended Data Fig. 3), for which the inflowing $B_{L}$ is all annihilated rather than reconnected in the EDR, was used in a benchmark test of the EMHD reconstruction code ${ }^{12}$.

Code availability. All code used to analyse the MMS data in this study is based on the publicly 
available SPEDAS tools ${ }^{46}$ (http://spedas.org/blog/), except for the Matlab code for the EMHD and polynomial reconstructions. The Matlab code for the EMHD reconstruction can be found at the Zenodo (https://doi.org/10.5281/zenodo.3900642), and that for the polynomial reconstruction at https://doi.org/10.5281/zenodo.3906853.

Data availability. All MMS data used in this study are publicly available via the MMS Science Data Center at https://asp.colorado.edu/mms/sdc/public/. Geomagnetic Auroral Electrojet index is available at http://wdc.kugi.kyoto-u.ac.jp/aedir/.

29. Torbert, R. B. et al. The FIELDS instrument suite on MMS: scientific objectives, measurements, and data products. Space Sci. Rev. 199, 105-135 (2016).

30. Lindqvist, P.-A., Olsson, G., Torbert, R. B. et al. The Spin-plane Double Probe electric field instrument for MMS, Space Sci. Rev., 199, 137-165 (2016).

31. Ergun, R. E., Tucker, S., Westfall, J. et al. The axial double probe and fields signal processing for the MMS mission, Space Sci. Rev., 199, 167-188 (2016).

32. Russell, C. T., Anderson, B. J., Baumjohann, W. et al. The Magnetospheric Multiscale Magnetometers, Space Sci. Rev., 199, 189-256 (2016).

33. Pollock, C. et al. Fast plasma investigation for Magnetospheric Multiscale. Space Sci. Rev. 199, 331-406 (2016).

34. Denton, R. E., Sonnerup, B. U. Ö., Russell, C. T. et al. Determining L-M-N current sheet coordinates at the magnetopause from Magnetospheric Multiscale data. J. Geophys. Res. Space Physics, 123, 2274-2295 (2018).

35. Sonnerup, B. U. Ö. \& Scheible, M. in Analysis Methods for Multi-Spacecraft Data (eds Paschmann, G. \& Daly, P. W.) Ch. 8, 185-220 (ISSI/ESA, 1998).

36. Shi, Q. Q., Tian, A. M., Bai, S. C. et al. Dimensionality, coordinate system and reference frame for analysis of in-situ space plasma and field data. Space Sci. Rev., 215, 35 (2019).

37. Shi, Q. Q., Shen, C., Dunlop, M. W. et al. Motion of observed structures calculated from multi-point magnetic field measurements: Application to Cluster. Geophys. Res. Lett., 33, L08109 (2006).

38. Denton, R. E., Sonnerup, B. U. Ö., Hasegawa, H. et al. Motion of the MMS spacecraft relative to the magnetic reconnection structure observed on 16 Oct 2015 at 1307 UT. Geophys. Res. Lett., 43, 5589-5596 (2016).

39. Kuznetsova, M. M., Hesse, M., Rastaetter, L. et al. Multiscale modeling of magnetospheric reconnection, J. Geophys. Res., 112, A10210 (2007).

40. Hasegawa, H., Sonnerup, B. U. Ö., Denton, R. E. et al. Reconstruction of the electron diffusion region observed by the Magnetospheric Multiscale spacecraft: First results. Geophys. Res. Lett., 44, 4566-4574 (2017).

41. Hasegawa, H., Denton, R. E., Nakamura, R. et al. Reconstruction of the electron diffusion region of magnetotail reconnection seen by the MMS spacecraft on 11 July 2017. $J$. Geophys. Res. Space Physics, 124, 122-138 (2019).

42. Nagai, T., Shinohara, I., Fujimoto, M. et al. Geotail observations of the Hall current system: Evidence of magnetic reconnection in the magnetotail. J. Geophys. Res., 106, 25929-25949 (2001).

43. Øieroset, M., Phan, T. D., Fujimoto, M., Lin, R. P., \& Lepping, R. P. In situ detection of collisionless reconnection in the Earth's magnetotail. Nature, 412, 414-417 (2001).

44. Torbert, R. B., Dors, I., Argall, M. R. et al. A new method of 3-D magnetic field reconstruction. Geophys. Res. Lett., 47, e2019GL085542 (2020).

45. Liu, Y.-H., Hesse, M., Guo, F. et al. Why does steady-state magnetic reconnection have a maximum local rate of order 0.1? Phys. Rev. Lett., 118, 085101 (2017).

46. Angelopoulos, V., Cruce, P., Drozdov, A., Grimes, E. W. et al. The Space Physics Environment Data Analysis System (SPEDAS). Space Sci. Rev., 215, 9 (2019). 
47. Rezeau, L., Belmont, G., Manuzzo, R., Aunai, N. \& Dargent, J. Analyzing the magnetopause internal structure: New possibilities offered by MMS tested in a case study. J. Geophys. Res. Space Physics, 123, 227-241 (2018).

48. Kawano, H. \& Higuchi, T. The bootstrap method in space physics: Error estimation for minimum variance analysis. Geophys. Res. Lett., 22, 307-310 (1995). 


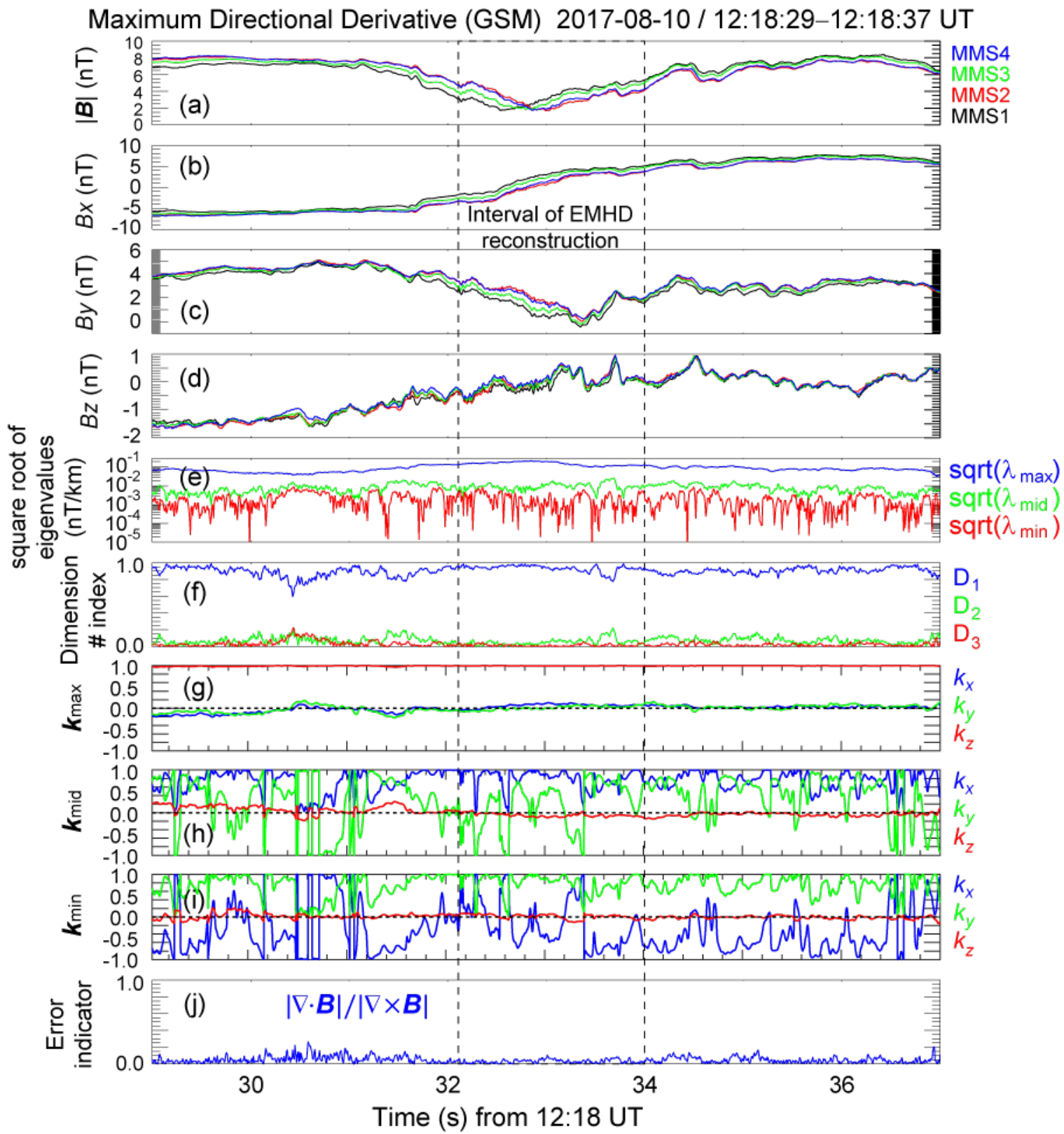

518 12:18:37 UT. a-d, GSM components of the magnetic field measured by the four MMS 519 spacecraft $\left(\mathbf{a} ;|\boldsymbol{B}|, \mathbf{b} ; B_{x}, \mathbf{c} ; B_{y}, \mathbf{d} ; B_{z}\right)$. e, Eigenvalues $\left(\lambda_{\max }, \lambda_{\operatorname{mid}}, \lambda_{\min }\right)$ of the $3 \times 3 \mathrm{MDD}$ 520 matrix $^{36}$. f, Dimension number indices ${ }^{47}$, defined as $D_{1}=\left(\lambda_{\text {max }}-\lambda_{\text {mid }}\right) / \lambda_{\max }, D_{2}=$ $\left(\lambda_{\text {mid }}-\lambda_{\text {min }}\right) / \lambda_{\text {max }}$, and $D_{3}=\lambda_{\text {min }} / \lambda_{\text {max }}$, that can be used as measures of the dimensionality of the structure encountered by the spacecraft, $. \mathbf{g}-\mathbf{i}$, Eigenvectors corresponding to the three eigenvalues ( $\left.\mathbf{g} ; \boldsymbol{k}_{\text {max }}, \mathbf{h} ; \boldsymbol{k}_{\text {mid }}, \mathbf{i} ; \boldsymbol{k}_{\text {min }}\right) . \mathbf{j},|\nabla \cdot \boldsymbol{B}| /|\nabla \times \boldsymbol{B}|$ as an error proxy. 
(a) Magnetic field lines MMS3 12:18:32.1-12:18:34.0 UT
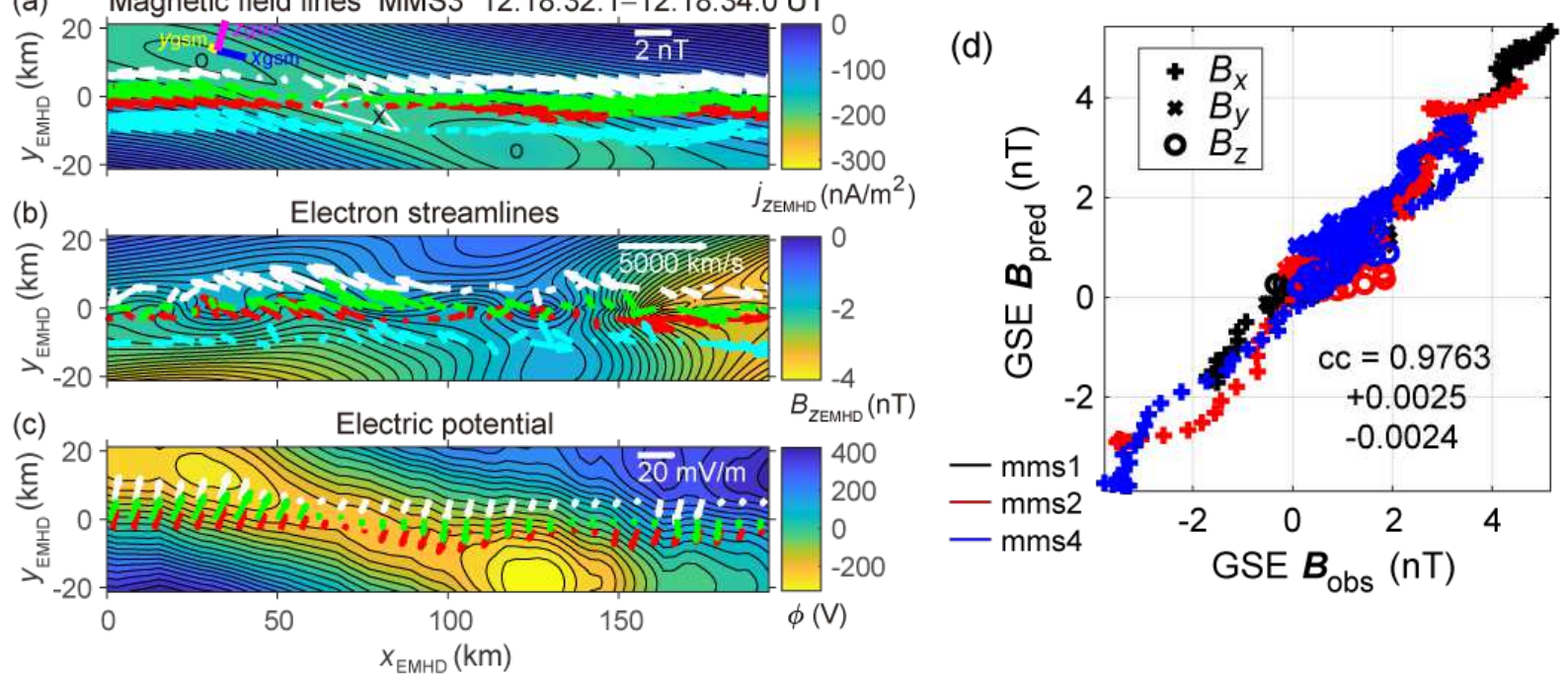

(d)

Extended Data Fig. 2 | 2-D magnetic, electrostatic and electron-velocity fields recovered from the EMHD reconstruction applied to the MMS 3 data at 12:18:32.1-12:18:34.0 UT. a, Reconstructed in-plane magnetic field-lines with the out-of-plane component $\left(j_{z, \text { EMHD }}\right)$ of the reconstructed current density in colour. Arrows show the projections onto the reconstruction plane of the measured magnetic fields (white, MMS 1; red, MMS 2; green, MMS 3; cyan, MMS 4) along the paths $\left(y_{\mathrm{EMHD}}=\right.$ const. $)$ of the four spacecraft. $\mathbf{b}$, Reconstructed electron streamlines with the out-of-plane component ( $\left.B_{z, \mathrm{EMHD}}\right)$ of the reconstructed magnetic field in colour. Arrows show the projections of the measured electron velocities transformed into the structure-rest frame. c, Reconstructed electrostatic potential $(\phi)$, along with the projections of the measured electric fields $\left(\boldsymbol{E}_{\mathrm{str}}=\boldsymbol{E}+\boldsymbol{V}_{\mathrm{str}} \times \boldsymbol{B}\right)$ transformed into the structure-rest frame. $\mathbf{d}$, Correlation between the components in geocentric solar ecliptic (GSE) coordinates of the measured magnetic field ( $B_{x}$ : plus, $B_{y}$ : cross, and $B_{z}$ : circle) and those predicted from the reconstruction along the paths of MMS 1 (black), MMS 2 (red), and MMS 4 (blue) not used as input for the reconstruction. The confidence intervals of the correlation coefficients corresponding to \pm 1 sigma were estimated by the bootstrap method ${ }^{48}$. 

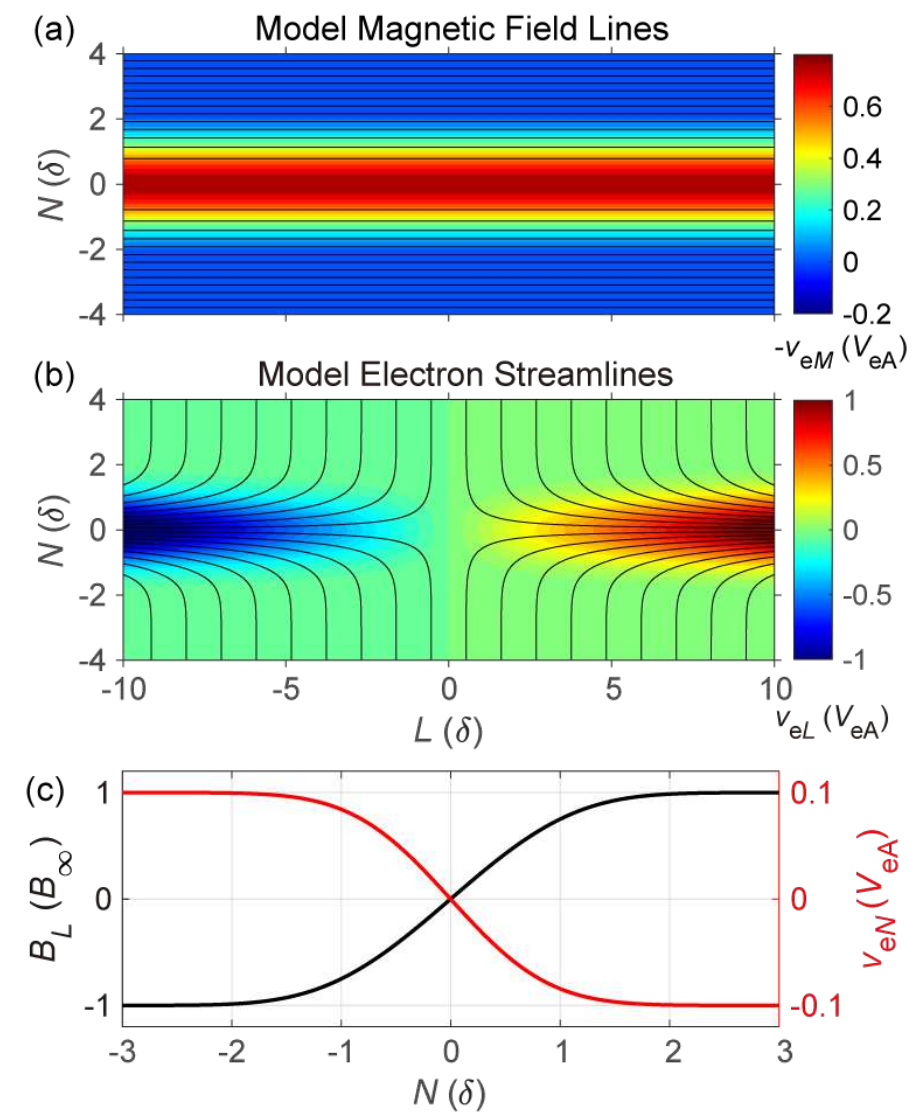

Extended Data Fig. 3 | Exact solution of the dissipative EMHD equations ${ }^{12}$ showing the magnetic field and electron velocity profiles in and around an ECS with a thickness $(\delta)$ comparable to $\boldsymbol{d}_{\mathbf{e}}$. a, Magnetic field-lines in the $L-N$ plane with the out-of-plane $(-M)$ component of the electron velocity in unit of $V_{\mathrm{eA}}$ in colour. $\mathbf{b}$, Electron streamlines with the $L$ component $\left(v_{\mathrm{e} L}\right)$ of the electron velocity in colour. c, Spatial profiles along $N$ of $v_{\mathrm{e} N}$ and $B_{L}$ in unit of the field intensity $\left(B_{\infty}\right)$ outside of the ECS. 

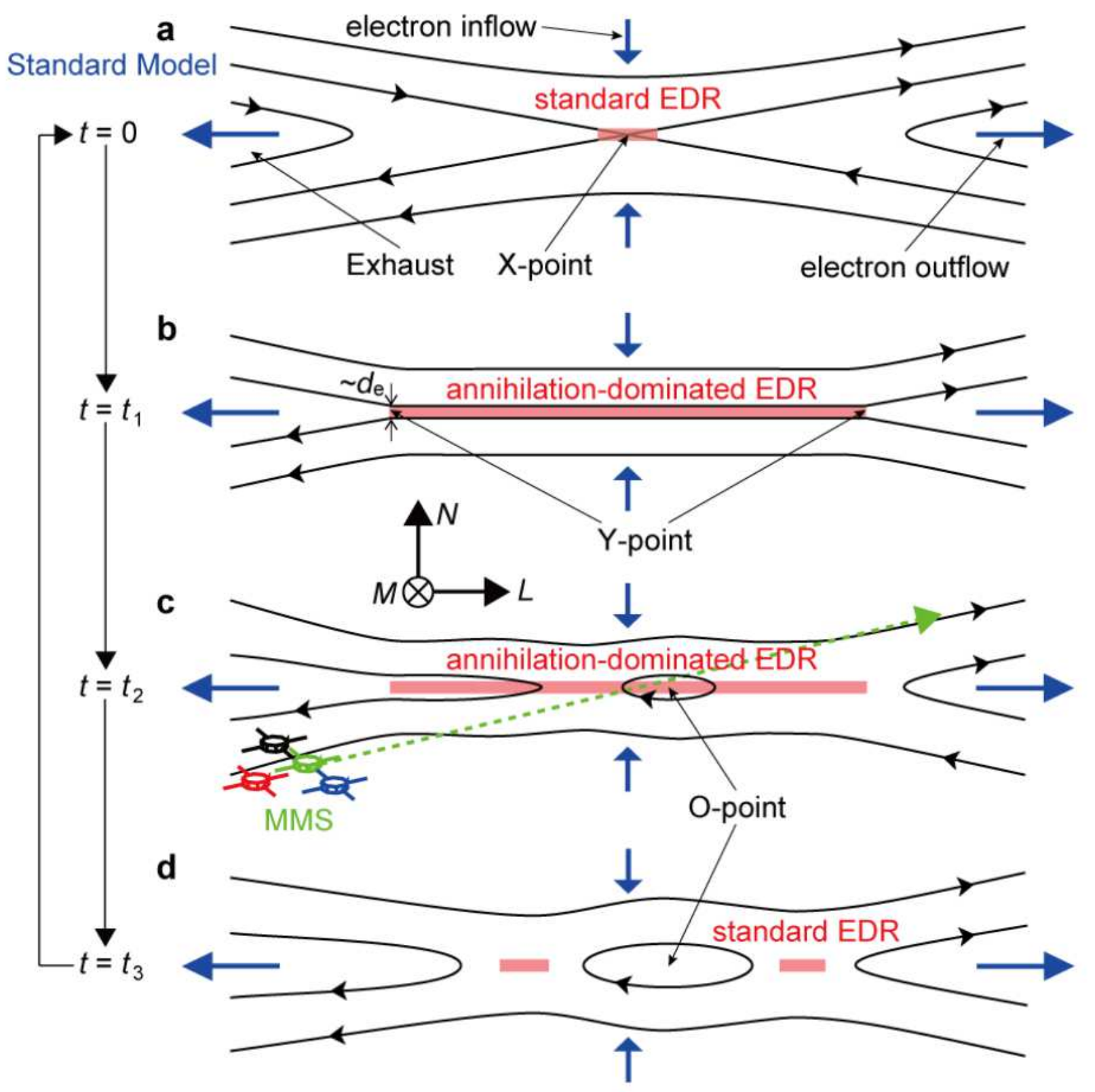

\section{Figure 1}

Standard picture of the reconnection region and possible sequence of time evolution of the observed electron-scale current sheet. a, Standard EDR with an X-type magnetic-field geometry where the magneticto-particle energy conversion is mostly due to reconnection4. b, EDR after elongation along $L$, as seen in simulation7, where the energy conversion may be mostly due to magnetic-field annihilation (Methods). c, Initial stage of the magnetic-island formation in the EDR, as observed by MMS. d, Possible later stage of the island growth in exhausts of the EDRs where the energy conversion is again dominated by reconnection. 


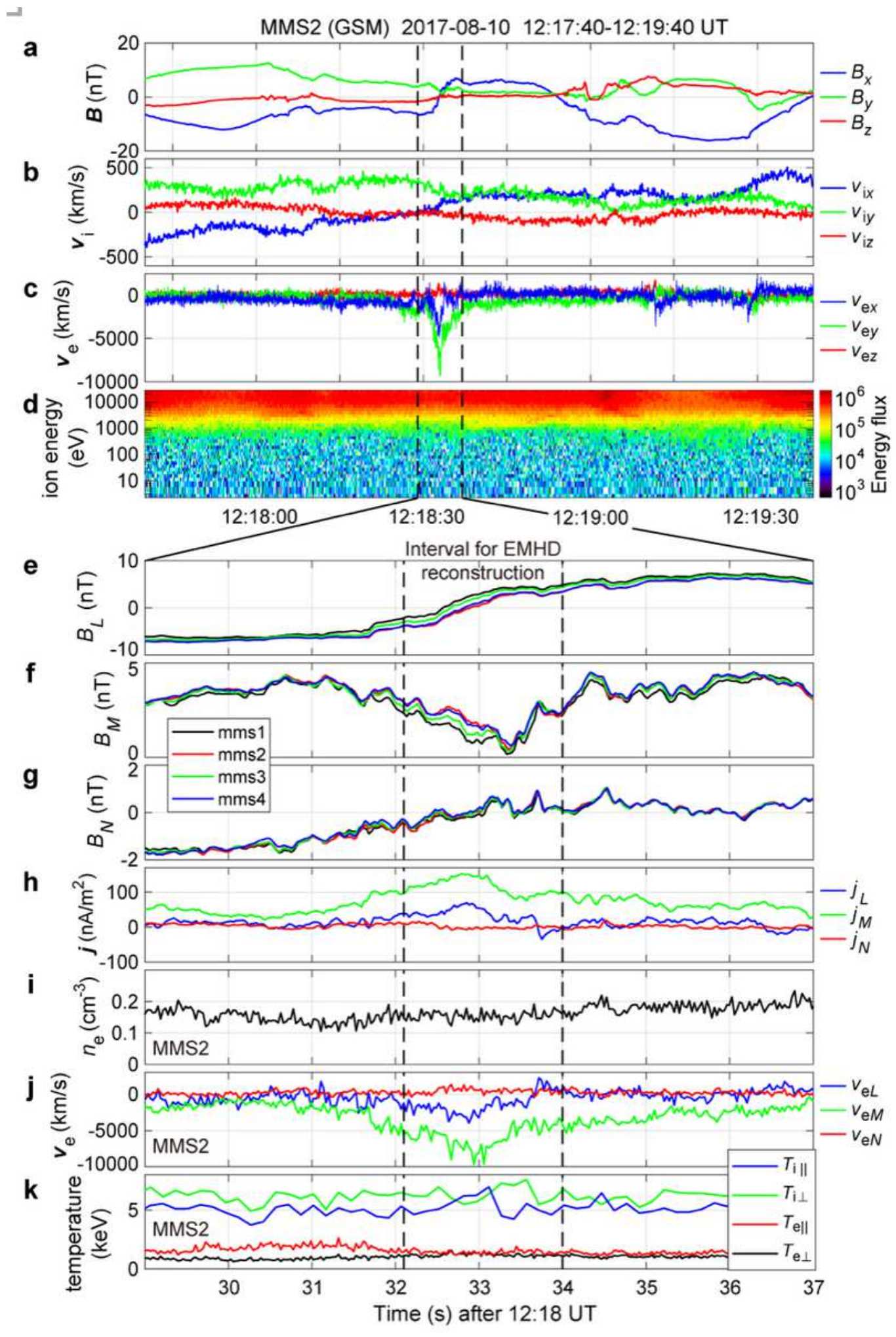

Figure 2

Overview of MMS observations of an ECS with both ion and electron reconnection jet signatures. a-d, GSM components of the magnetic field ( $a$; Bx, By and Bz), ion velocity (b; vix, viy and viz), electron velocity (c; vex, vey and vez) and ion energy-time spectrogram of omni-directional differential energy flux (d; colour scale, in units of keV s-1 cm-2 sr-1 keV-1) seen by MMS 2. e-h, Components in a common current-sheet (LMN) coordinate system of the magnetic field (e-g; BL, BM and $B N$ ) from all four 
spacecraft (black, MMS 1; red, MMS 2; green, MMS 3; blue, MMS 4) and current density j=( $(\times \mathrm{B}) / \mu 028$ (h; jL, jM and jN). i-k, MMS 2 measurements of the electron density (i; ne), electron velocity in LMN coordinates (j; veL, veM and veN), and ion and electron temperatures ( $k$; Ti and Te) in the directions parallel $(\nabla)$ and perpendicular $(\rrbracket)$ to the local magnetic field.

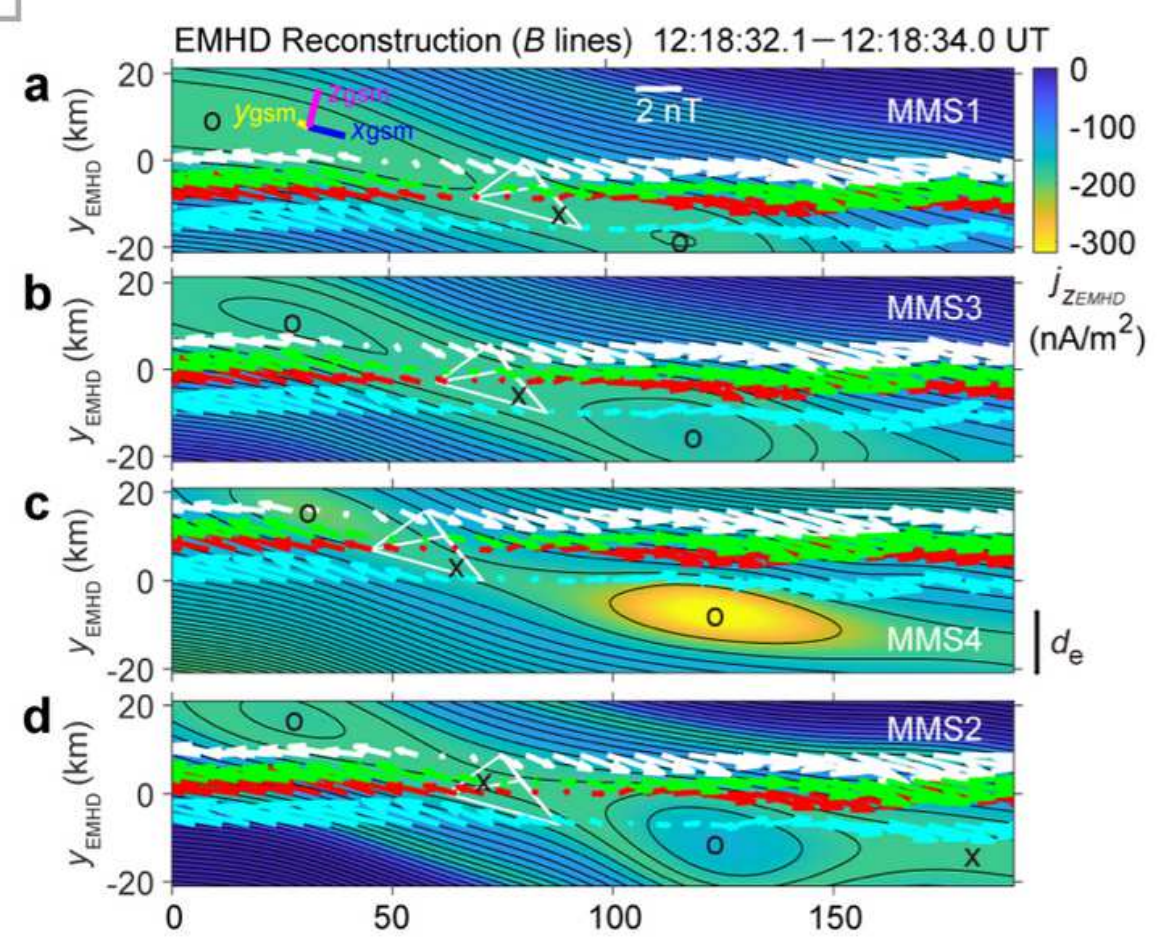

Time after Polynomial Reconstruction

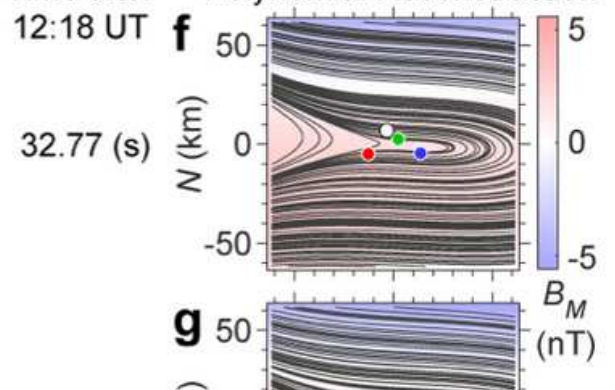

$32.8(\mathrm{~s})$
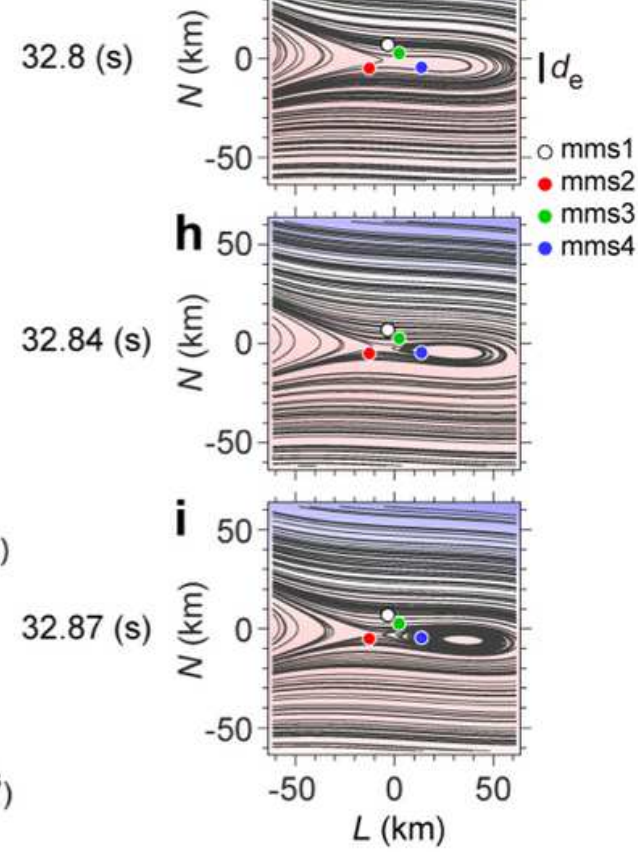

\section{Figure 3}

Magnetic fields recovered from the two reconstruction methods (Methods). a-d, 2-D magnetic fields from the EMHD reconstruction12 using the data taken at 12:18:32.1-12:18:34.0 UT individually for each of the four spacecraft, shown in the order of current sheet crossing (MMS 1, a; MMS 3, b; MMS 4, c; then MMS 2, $\mathrm{d}$; as seen in Fig. 2e). Black curves show the reconstructed magnetic field-lines, colours the out-of-plane component (jz,EMHD) of the reconstructed current density, and arrows the projections onto the reconstruction (xEMHD-yEMHD) plane of the measured magnetic fields (white, MMS 1; red, MMS 2; green, MMS 3; cyan, MMS 4) along the paths of the four spacecraft. The bars near the upper-left corner of

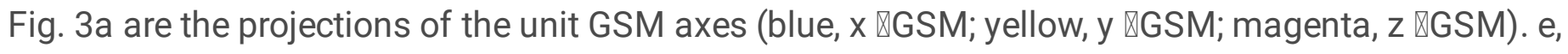
Magnetic field-lines (black curves) and electron streamlines (blue curves) reconstructed from the MMS 3 data, with $j \otimes E^{\prime}=j p \otimes(E+v e \times B) 14$ measured along the paths of MMS 1, MMS 2, and MMS 3 in colour. $f-i$, Projection onto the $\mathrm{L}-\mathrm{N}$ plane of 3-D magnetic field-lines reconstructed using the polynomial 

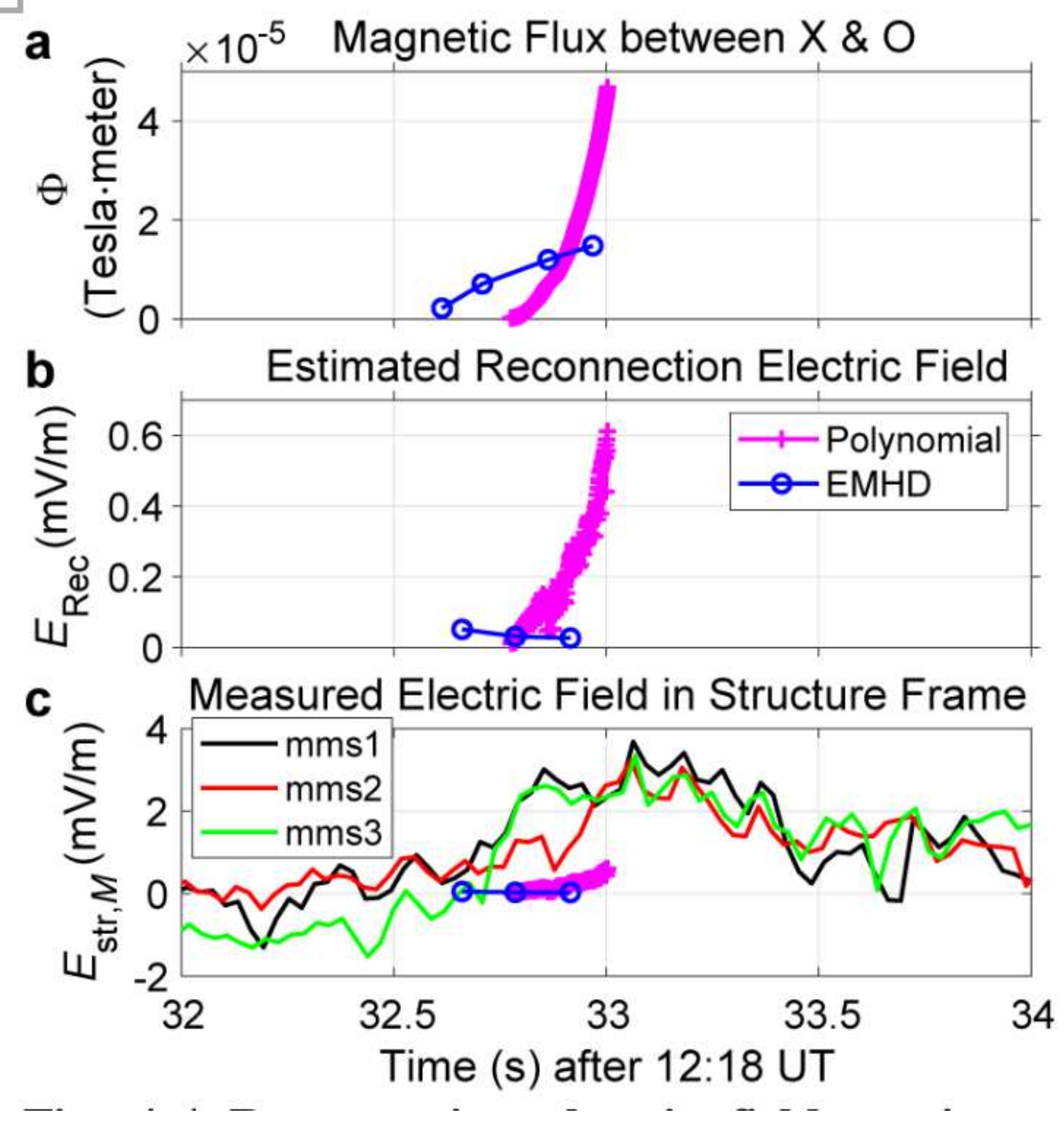

Figure 4

Reconnection electric fields, estimated from the EMHD and polynomial reconstructions, compared with the measured electric field. a, In-plane magnetic flux $(\Phi)$ embedded between the $X$ - and 0-points around the centre of the reconstruction domain as a function of time which is defined as that of the current sheet crossing $(B L=0)$ for the $E M H D$ reconstruction. $b$, Reconnection electric field $(E R e c=\partial \Phi / \partial t)$ estimated from the temporal rate of change in $\Phi$. c, M component (Estr,M) of the electric field from MMS 1, MMS 2, and 
MMS 3 that made reliable electric field measurements, transformed into the frame comoving with the structure (Methods), along with ERec. 